\title{
Performance comparison of a silica gel-water and activated carbon-methanol two beds adsorption chillers
}

\author{
Adam Szelągowski ${ }^{1, *}$, and Andrzej Grzebielec ${ }^{1}$ \\ ${ }^{1}$ Institute of Heat Engineering, Faculty of Power and Aeronautical Engineering, Warsaw University \\ of Technology, Poland
}

\begin{abstract}
The aim of the study is to compare the efficiency of adsorption refrigerating equipment working with different working pairs. Adsorption cooling devices can operate with a relatively low temperature of heat sources while consuming only a small amount of electricity for the operation of auxiliary equipment. Refrigerants used in adsorption devices are substances that do not have a negative impact on the environment. All that makes that adsorption refrigeration seems to be a good solution for utilizing renewable and waste heat sources for cold production. To carry out the experiment the adsorption cooling device has been developed and researched in Institute of Heat Engineering at Warsaw University of Technology. The test bench consisted of two cylindrical adsorbers, condenser, evaporator, oil heater and two oil coolers. In order to perform the correct action it has been developed and implemented special control algorithm device, allowed to keep the temperature in the evaporator at a preset level. The unit tested for two sorption pairs: activated carbon - methanol, and silica gel - water. For activated carbon - methanol working pair it was obtained energy efficiency rating (EER) equals to 0.14 and specific cooling power (SPC) of $16 \mathrm{~W} / \mathrm{kg}$. For silica gel - water EER of refrigeration unit was 0.25 and SPC was equal to $208 \mathrm{~W} / \mathrm{kg}$.
\end{abstract}

\section{Introduction}

Solid sorption is used in many applications including thermally driven refrigeration technologies. Solid sorption or adsorption refers to processes where a vapor is taken up by a solid when both the gas and the solid are in contact. Vapour molecules are accumulated on the surface of the solid and remain attached to it [1]. This process can be reversed and the adsorbed phase can be released from the adsorbent by applying heat to the compound. Adsorption refrigeration covers two processes, which are the heating-desorbing process and the cooling-adsorbing process. Because of that the simple traditional cycle is a type of intermittent refrigeration cycle [2]. If the heat source can be provided continually and the continuous refrigeration effect is required, two adsorber bed or multi adsorber beds device need to be designed for an adsorption refrigeration system, for which the heating and

*Corresponding author: adam.szelagowski@itc.pw.edu.pl 
cooling processes of multi adsorbers will be complementarily arranged [3]. Adding every extra adsorber to device makes the cooling effect of the device is more stable, but it is associated with more complex and more difficult controlling devices work. Because of those reasons the most common adsorption units with continuous cooling effect contain two beds [4]. Figure 1 shows thermodynamics states of adsorbers for two bed unit during the operation. During the first half-cycle adsorber 1 is being heated, while adsorber 2 is being cooled, during the second half-cycle adsorber 1 is being cooled, while adsorber 2 is being heated [5]. During the first phase of working process (line A-B in Fig. 1), adsorber absorbs applied heat thus increasing the adsorbent's temperature which induces a pressure increase from evaporation pressure to condensation pressure. This phase is also known as isosteric heating [5]. The temperature of adsorbent bed increases from $t_{A}$ to $T_{B}$. After this vapours of refrigerant are released from the bed, and they move towards condenser where they cool down and condense. While adsorber receiving heat continues but being connected to condenser, desorption of vapor get induced and liquefaction of desorbed vapor in condenser further takes place (line B-C in Fig. 1). The temperature of the adsorbent bed increases from $t_{B}$ to $t_{C}$, but pressure stays at condensing pressure level. This phase is also known as isobaric desorption [5]. The liquid refrigerant flows through expansion value and evaporated in the evaporator providing cooling power [6]. Meanwhile, temperature of second adsorbent reduces due to release of heat (line C-D in Fig. 1) which induces pressure decrease from condensation pressure to evaporation pressure. This phase is also known as isosteric cooling [5]. The pressure get reduced from condensation pressure $p_{c}$ to evaporation pressure $p_{e}$ and temperature also decreases to $t_{D}$. The adsorbent temperature continues to reduce due to further release in heat which causes adsorption of vapor due to connection with evaporator. The adsorbent is cooled from $t_{D}$ to $t_{A}$, pressure stays at evaporation level. This phase is also known as isobaric adsorption [5]. The whole process of adsorption cooling requires only heat energy which can be supplied through gas or oil fired or solar energy [7]. Unlike absorption processes, adsorption cooling systems have the advantage, that can be driven by lower temperature of heat source [8]. Driving heat source, can be at such low temperature as $50^{\circ} \mathrm{C}$. Therefore, solar radiation, waste heat, as well as geothermal energy can be used to power refrigeration unit systems. Using solar energy, as a free and renewable type of energy, to drive adsorption cooling systems is considered an attractive option as well as a focal point of interest. Adsorption cooling systems can be also used in cogeneration systems [9]. Refrigerant used as a working fluid in adsorption cooling devices usually do not have a negative impact on the environment. In conjunction with the use of waste and renewable energy sources, application of these devices use introduce a good environmental and social factor [10]. Selection of the working pair is essential to adjust the application. In application where cooling temperature can be above $0^{\circ} \mathrm{C}$ water can be used as a refrigerant with any adsorbents. For applications below $0^{\circ} \mathrm{C}$ methanol, ethanol or ammonia with activated carbon can be used [11]. Multi bed devices allow to improve efficiency of the unit by recovery the heat between the adsorbers. Such solution can improve the efficiency of the whole system by about $30 \%$. Adsorption units that work in basic continuous cycle have large possibility to recover heat from one adsorber to another. In the beginning phase of the process the first adsorber has to be cooled, when second one has to be heated. Temperature difference, between point $\mathrm{C}$ and A (Fig. 1) is large enough to transport heat from one bed to another. Creating such heat recovery system in the beginning phase of process the external heat source and external cooling system is not needed. 


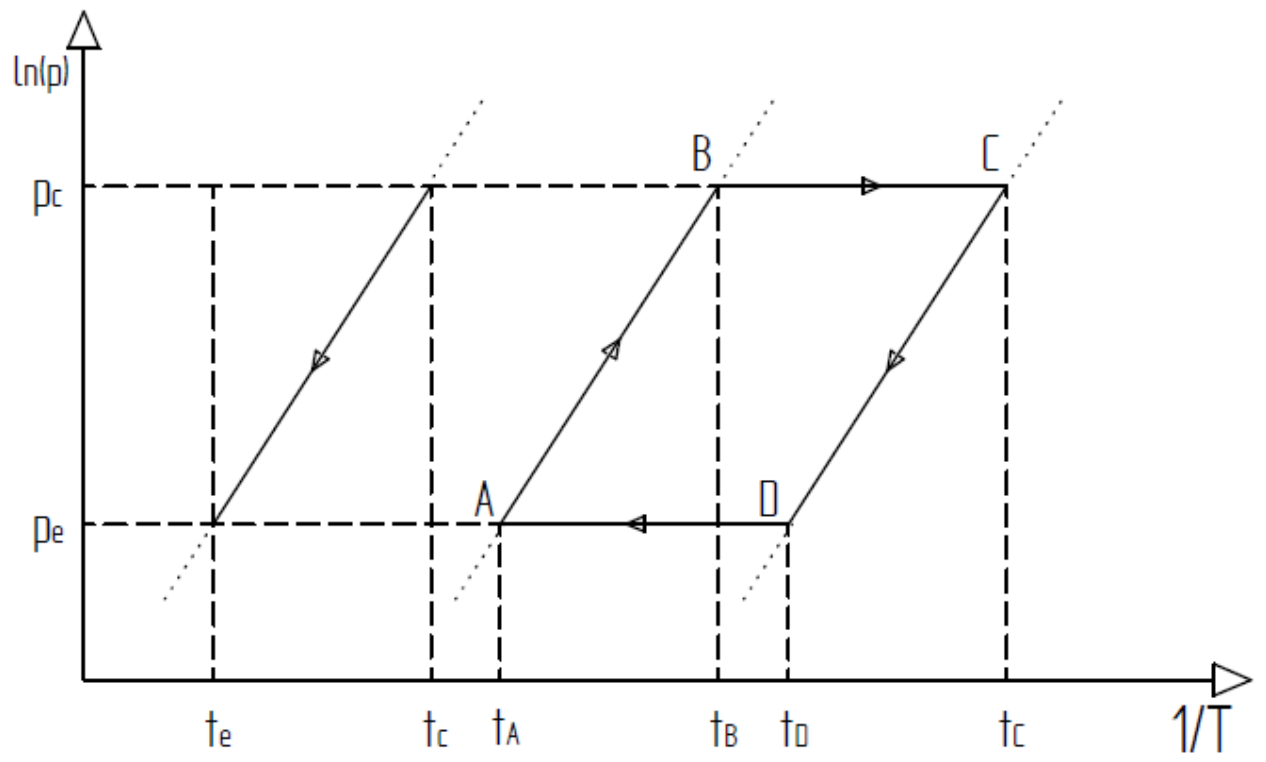

Fig. 1. Thermophysics states of adsorpbers during the operation.

\section{Construction of the apparatus}

The thermal wave adsorption refrigeration device was designed and built at the laboratory of the Division of Refrigeration and Energy in Buildings (formerly Division of Processes Equipment and Cooling), Faculty of Power and Aeronautical Engineering, Warsaw University of Technology. The thermal wave device allows to recover heat between adsorber beds during the process [12]. When the direction of oil circulation is reversed, the secondary fluid recovers the heat from one adsorber and transfers it to the second one. The installation is designed to examine this device comprises three circulation systems: heat transfer fluid, refrigerant and heat sink. Figure 2 shows an overview of the apparatus. The adsorber consists of an inner brazen tube with $12 \mathrm{~mm}$ outer diameter, through which the heat transfer medium flows, supplying the heat to the bed or taking the heat back from it. The inner tube is mounted inside an outer tube with $28 \mathrm{~mm}$ out diameter. Between the tubes there is a sorption material bed. In the centre of the inner tube a pipe is soldered, through which the refrigerant flows to and from the bed. The whole assembly is housed in a tight foam insulation. The heat flux should occur between the adsorbent bed and the heat transfer medium rather than between the bed and the air surrounding the adsorber. Device was tested with two working pairs. In one configuration, the methanol as refrigerant and activated carbon as sorption material were selected as the working pair. In the second configuration, the water as refrigerant and silicagel as sorption material were selected. 


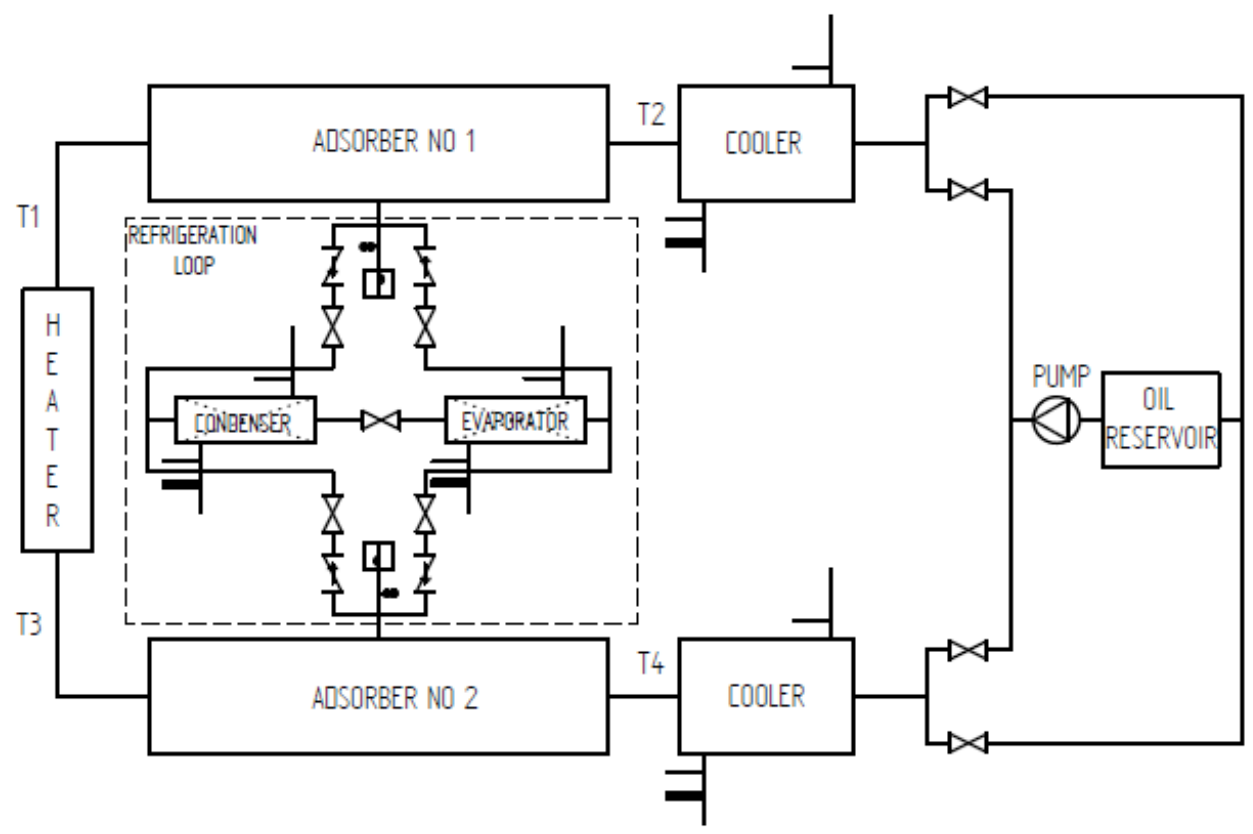

Fig. 2. Thermal wave adsorption refrigeration device.

\subsection{Secondary fluid circulation}

The heat is transferred to and from the adsorber beds by the mineral oil circulation system. Oil system comprises a circulating pump, oil surge tank, five heat exchangers consisting of two coolers, two adsorber beds and one electric heater (Fig. 2). An assembly of four solenoid valves was mounted to control the direction of oil flow. In the first half cycle oil is pumped from surge tank through first cooler, first adsorber bed, than through the electric heater to the second adsorber bed. Downstream oil flows through second cooler to the surge tank. In the second half cycle, the oil flows in the opposite direction. The open surge tank protects the system against an uncontrolled pressure rise, and compensates for thermal expansion of the working fluid.

\subsection{Refrigerant circulation}

The refrigerant circulates in the system between adsorber beds, condenser and evaporator. The refrigerant circulation system comprises a set of solenoid valves and in case of methanol non-return valves which provide a connection between the adsorber beds on one side and the condenser and evaporator on the other. Each adsorber bed is connected with condenser and evaporator (Figure 2). This set and proper control of the opening of the valves makes the stream of refrigerant from the desorbing bed always flow to the condenser, and then, after flowing through the electronic expansion valve, to the evaporator. Finally, the vapor refrigerant is adsorbed by the second adsorber bed. Initialization and opening time for valves are determined on the pressure values in the adsorbers. 


\subsection{Heat sink circulation}

To dissipate heat from the system heat sink circulation is required. In order to determine heat exchanger capacities, a water circulation system was introduced. The water flows through condenser, evaporator and both heat sink exchangers. All capacities in mentioned heat exchangers are calculated according to Equation 1.

\section{Methodology}

In the context of experimental studies a continuous oil temperature were measured at inlet and outlet of adsorbers (Fig. 2). There was also measured temperature of the water before and after all heat exchangers through which water flows. Based on these parameters instantaneous heat capacities have been determined according to the equation 1 :

$$
\dot{Q}=\dot{m} \cdot c_{p} \cdot\left(T_{\text {out }}-T_{\text {in }}\right)
$$

Mass flow $\dot{m}$ was determined on the volumetric flow rate $\dot{V}$ according to the equation 2 :

$$
\dot{m}=\dot{V} \cdot \rho
$$

The oil volumetric flow rate $\dot{V}$ was determined by measuring the known volume filling time. Volume flow rate was measured during flow in both direction. In both cases, the measured value eqals $\dot{V}=1.7 \mathrm{l} / \mathrm{min}$. For the temperature measuring oil density was $\rho=880 \mathrm{~kg} / \mathrm{m}^{3}$.

For temperature measurements were used NTC5 temperature sensors with measurement uncertainty of $1 \%$. Filling time was measured with accuracy of $\pm 1 \mathrm{~s}$ and the tank volume was 2 liters. During experiment, there was measured pressure of refrigerant in the adsorbers with piezoelectric quartz pressure transducer having an accuracy of measurement of $\pm 0.3 \%$. Based on the pressure $P$, there was determined saturation temperature $T$ for evaporation and condensation in accordance to the saturation line equation [13]:

$$
\ln P=a+\frac{b}{T}
$$

Where the coefficients $a$ and $b$ for methanol are $\mathrm{a}=20.84$ and $\mathrm{b}=-4694$. For water $a=20.5896$ and $b=-5098.26$. In the equation 3, the temperature is given in Kelvin degrees and the pressure is calculated in mbar. The temperature determined from the measured pressure determines the heat source requirements the adsorption device can co-operate.

The energy efficiency rate EER of adsorption refrigeration devices represents amount of coolness generated in evaporator to amount of heat generated in heat source ratio. It can be calculated from the equation (4)

$$
\operatorname{EER}=\frac{Q_{C}}{Q_{H s}}
$$

wherein $Q_{C}$ is the amount of heat obtained in evaporator during one half cycle according to equation 5. $Q_{H S}$ is the amount of heat supplied to the system at the same time by heat source according to the equation 1 and trapezoidal integration method (equation 6).

$$
Q_{C}=m_{a d s}\left(w_{\max }-w_{\min }\right) h_{f g}
$$


In equation (5) $m_{a d s}$ is mass of adsorbent in a single bed $\left[\mathrm{kg}_{\text {adsorbent }}\right]$, $w_{\max }$ is maximum uptake of refrigerant in adsorbent $\left[\mathrm{kg}_{\text {refrigerant }} / \mathrm{kg}_{\text {adsorbent }}\right], w_{\text {min }}$ is minimum uptake of refrigerant in adsorbent, $h_{f g}\left[\mathrm{~J} / \mathrm{kg}_{\text {refrigerant }}\right]$ is specific latent heat of vaporization of refrigerant.

$$
Q_{H S}=\int_{t_{0}}^{t_{1}} \dot{Q}_{H S}(t) d t
$$

wherein $t_{0}$ is half cycle start time, and $t_{l}$ is time of half cycle period. $\dot{Q}_{H S}$ is the heat flux supplied to the system during selected time step.

\section{Research results}

The pressure value in each adsorber was measured and it was used to operate the electromagnetic valves that control the refrigerant and oil flow direction. A pulse type electronic expansion valve was used, so that the pressure and evaporation temperature could be controlled using researchers' software. The boiling point of both tested refrigerants (methanol, water) was set to be $10^{\circ} \mathrm{C}$. Fig. 3 and 4 show heat fluxes during four full half cycle, during the further course of the experiment the results are reproducible and characteristics are like those presented.

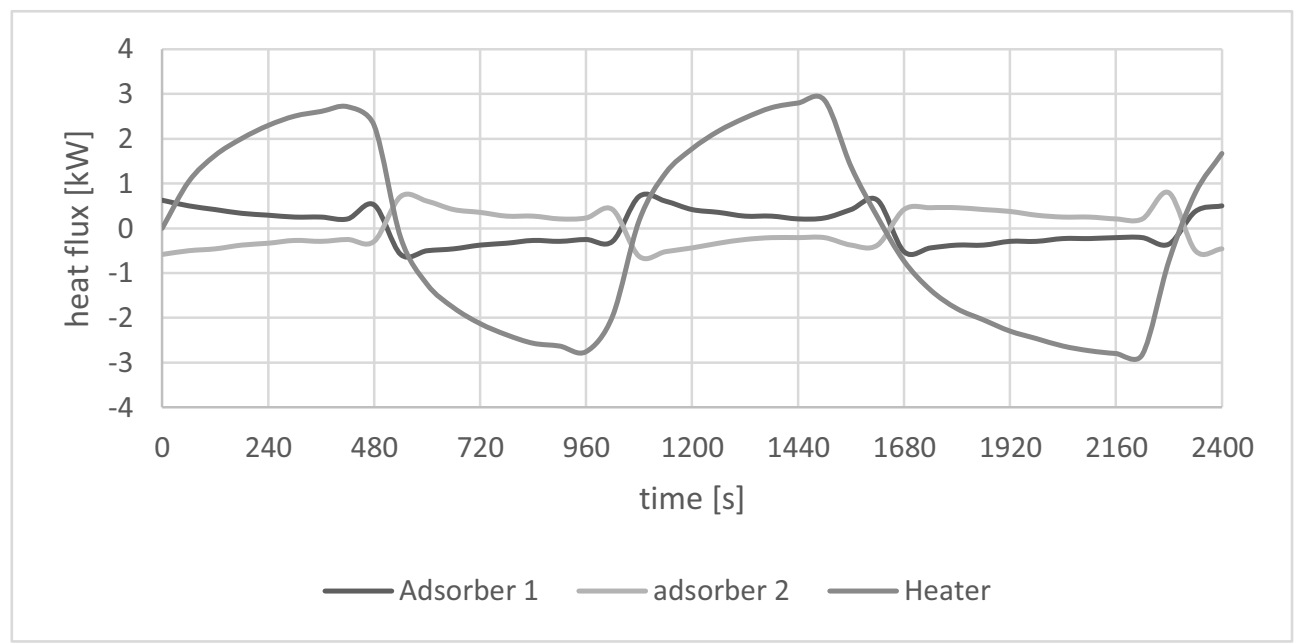

Fig. 3. Heat fluxes provided / received for component for activated carbon - methanol working pair. 


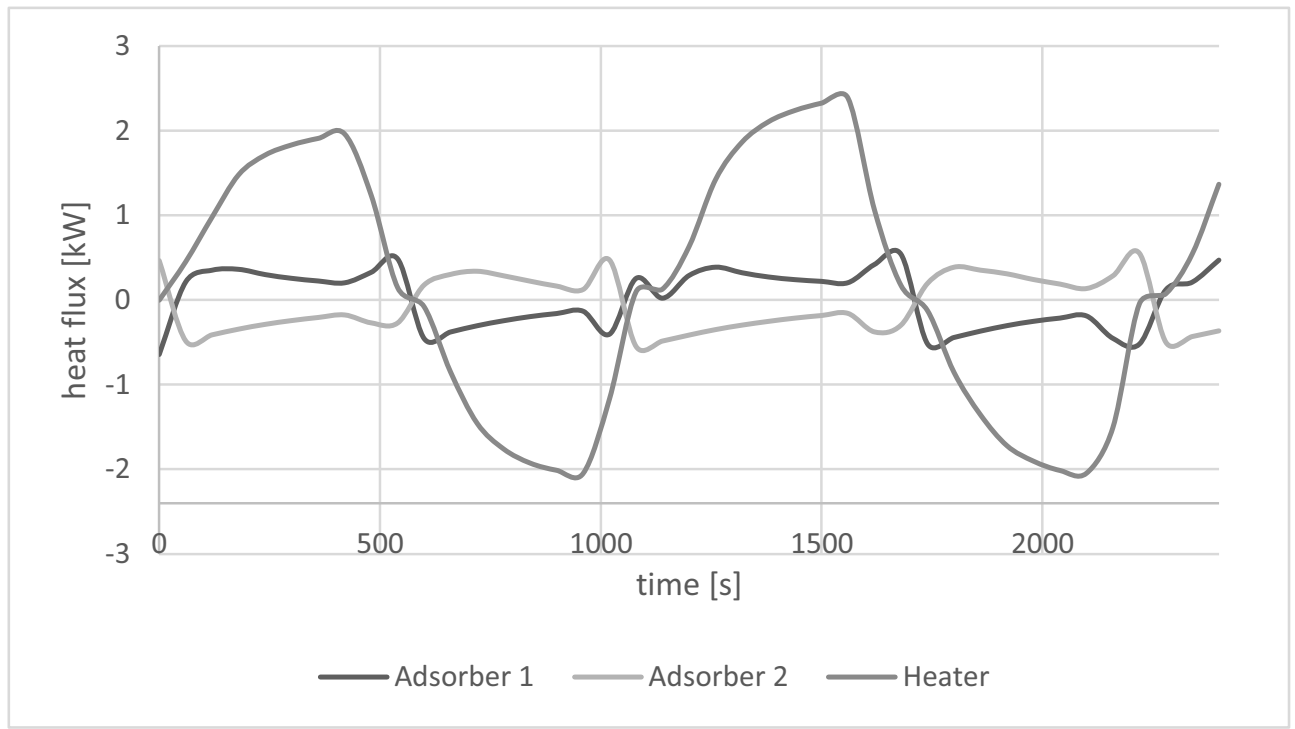

Fig. 4. Heat fluxes provided / received for component for silica gel - water working pair.

In the first half-cycle the temperature difference between $\mathrm{T} 1$ and $\mathrm{T} 2$ represents heat of desorption while the temperature difference between T3 and T4 corresponds to heat of adsorption in the second adsorber. Based on the measurements, real instantaneous powers of each component of the system could be determined. The energy efficiency rating EER of adsorption devices is determined based on Eq. (4). With measurements of instantaneous thermal power, the amount of heat can be determined from the integrals of the power over the time. What has been done here by trapezoids. For investigated case the average value of EER was obtained at level 0.14 for activated carbon - methanol and 0.25 for silica gel - water. Specific cooling power (SPC) was $16 \mathrm{~W} / \mathrm{kg}$ for activated carbon - methanol and $208 \mathrm{~W} / \mathrm{kg}$ for silica gel - water.

\section{Summary}

In this article the results of the experimental research concerning a state-of-the-art thermal wave adsorption refrigeration device are presented. Due to different working temperatures of the adsorbers, the change in the thermal power of each system component could be determined. The measurement results are presented in Figures 3 and 4 show four half cycles of the adsorption device operating under steady state conditions. In the following experiment, the cycle is repeated, so that these results can be regarded as representative for the calculation of the energy efficiency rating of the adsorption refrigeration apparatus.

There was energy efficiency rating (EER) obtained at level 0.14 for activated carbon - methanol and 0.25 for silica gel - water working pair. Specific cooling power (SPC) was $16 \mathrm{~W} / \mathrm{kg}$ for activated carbon - methanol and $208 \mathrm{~W} / \mathrm{kg}$ for silica gel - water. Low energy efficiency was due to the excessive duration of the half cycles representing approximately 6 minutes.

\section{References}

1. A. Ruciński, A. Rusowicz, K. Rucińska Przem. Chem. 1, 7 (2016) 
2. H. Z. Hassan, A. A. Mohamad, Y. Alyousef, H. A. Al-Ansary, Sustain. Energy Rev. 45 (2015)

3. H. Deshmukh, M. P. Maiya, S. Srinivasa Murthy, Appl. Therm. Eng. 82 (2015)

4. T. X. Li, R. Z. Wang, H. Li, Prog. Energy Combust. Sci., 40, 1 (2014)

5. P. Goyal, P. Baredar, A. Mittal, A. R. Siddiqui, Renew. Sustain. Energy Rev. 53 (2016)

6. W. Kuczyński, T. Bohdal, H. Charun, Exp. Heat Transf. 26, 1 (2013)

7. I. I. El-Sharkawy, H. AbdelMeguid, B. B. Saha, Appl. Energy 126 (2014)

8. A. Rusowicz, A. Ruciński, Environ. Eng. 1-3 (2011)

9. M. Jaworski, M. Bednarczyk, M. Czachor, Appl. Therm. Eng. 96 (2016)

10. P. Cyklis and K. Janisz, Chem. Process Eng- Inz, 36, 3 (2015)

11. A. Grzebielec, A. Rusowicz, A. Ruciński Przem. Chem. 1, 6 (2015)

12. A. Grzebielec, A. Rusowicz, R. Laskowski, Chem. Process Eng-Inz. 36, 2 (2015)

13. G. Cacciola, G. Restuccia, Int. J. Refrig. 18, 2 (1995) 